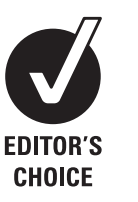

See Editorial, p 1265

${ }^{1}$ Department of Cardiology and Angiology, Adult Congenital and Valvular Heart Disease Center, University Hospital Muenster, Muenster, Germany

${ }^{2}$ Department of Thoracic and Cardiovascular Surgery, University Hospital Muenster, Muenster, Germany

Correspondence to Professor Helmut Baumgartner Adult Congenital and Valvular Heart Disease Center, Department of Cardiology and Angiology, University Hospital Muenster,

Albert-Schweitzer-Campus 1, Muenster 48149, Germany; helmut.baumgartner@ ukmuenster.de

Accepted 8 May 2012 Published Online First 11 June 2012

\title{
Impact of transcatheter aortic valve implantation or surgical aortic valve replacement on right ventricular function
}

\author{
Aleksander Kempny, ${ }^{1}$ Gerhard-Paul Diller, ${ }^{1}$ Gerrit Kaleschke, ${ }^{1}$ Stefan Orwat, ${ }^{1}$ \\ Angela Funke, ${ }^{1}$ Renate Schmidt, ${ }^{1}$ Gregor Kerckhoff, ${ }^{1}$ Farshad Ghezelbash, ${ }^{1}$ \\ Andreas Rukosujew, ${ }^{2}$ Holger Reinecke, ${ }^{1}$ Hans H Scheld ${ }^{2}$ Helmut Baumgartner ${ }^{1}$
}

\begin{abstract}
Objective Transcatheter aortic valve implantation (TAVI) has become an alternative to surgical aortic valve replacement (sAVR) in selected high risk patients. While improvement in left ventricular function after TAVI has been demonstrated, little is known about the impact on right ventricular (RV) function. Since postoperative RV dysfunction is linked to adverse outcomes, the authors sought to investigate the effect of TAVI and aortic valve replacement (AVR) on RV function using speckle tracking echocardiography.
\end{abstract}

Design Cross-sectional study in tertiary healthcare setting.

Setting 101 patients with severe symptomatic aortic stenosis (age $81 \pm 11 \mathrm{yrs}$ ) who underwent TAVI and 22 patients who underwent SAVR were included. RV function was assessed using 2D longitudinal strain (RVLS), fractional area change and tricuspid annular plain systolic excursion before and after sAVR and TAVI (median 89 days).

Results Although the TAVI group had worse baseline characteristics, RV function remained unchanged in this group whereas significant deterioration of RV function was observed in patients undergoing conventional AVR: RV-LS (-25.2 \pm 6.1 vs $-20.0 \pm 7.0 \% ; p=0.009)$,

RV-fractional area change $(47.0 \pm 7.0$ vs $39.8 \pm 10.7 \%$, $\mathrm{p}=0.019$ ) and tricuspid annular plain systolic excursion (24 \pm 5 vs $16 \pm 4 \mathrm{~mm}, p=0.0001$ ).

Conclusion While TAVI did not affect RV function it deteriorated significantly in patients undergoing SAVR. The authors speculate that this may be related to the detrimental effects of pericardiotomy and, to a lesser degree, cardiopulmonary bypass. While further studies are required to assess the clinical significance of this finding, these data suggest that patients with pre-existing RV dysfunction may benefit from TAVI and that RV function should be incorporated into future risk scores.

\section{INTRODUCTION}

Transcatheter aortic valve implantation (TAVI) has become an alternative to surgical aortic valve replacement (sAVR) in selected patients considered to be at a high risk for conventional cardiac surgery. Risk stratification is usually based on score systems such as the EuroSCORE or the Society of Thoracic Surgeons' risk model. ${ }^{1-3}$ Early results of TAVI have been published showing high procedural success and a low complication rate. ${ }^{4}$
While the impact of sAVR or TAVI on the function of left ventricle has been studied with various imaging modalities, little is known about the impact on right ventricular (RV) function. ${ }^{5-9}$ RV failure after cardiac surgery, although not frequent, has been linked to adverse outcome in this setting. ${ }^{10} 11$ The aetiology of postoperative RV dysfunction is likely multifactorial and related to factors such as pericardiotomy with perturbation of myocardial blood flow, hypothermia and immuneinflammation on cardiopulmonary bypass. Interestingly, both the EuroSCORE and the Society of Thoracic Surgeons' risk model do not account for preoperative RV dysfunction although it is one of the strongest predictors of RV failure after cardiac surgery. $^{2}{ }_{3} \quad 11 \quad 12$ Since TAVI does not require cardiopulmonary bypass or pericardiotomy, we hypothesised that it may have less detrimental impact on RV function. In the current study, we sought to assess the impact of sAVR and TAVI on the RV function using conventional echocardiographic parameters as well as speckle tracking echocardiography.

\section{PATIENTS AND METHODS \\ Patients}

We studied all 101 consecutive adult patients with severe aortic stenosis in whom TAVI was performed between February 2008 and May 2010 at our institution. The sAVR group consisted of 22 consecutive patients with adequate preoperative and postoperative echocardiograms in whom sAVR was performed at our institution between May 2008 and July 2009.

\section{Echocardiographic measurements}

Transthoracic echocardiography was performed prior to intervention (median time 19 days (IOR 7-32 days) in the TAVI group and 18 days (IOR 11-36 days) in the sAVR group) and after intervention (70 days (IOR 26-106 days) in the TAVI group and 100 days (IOR 68-117 days) in the sAVR group). With the subject in the left lateral position, transthoracic examinations were conducted according to current guidelines. ${ }^{13-15}$ Echocardiographic studies were performed in all subjects with a Vivid 7 Dimension system (Vingmed, General Electric, Milwaukee, Wisconsin, USA). All recordings were stored digitally and 
analysed offline. Tricuspid annular plane systolic excursions (TAPSE) were measured in the apical 4-chamber view using M-Mode echocardiography. TAPSE was defined as the maximal excursion at the lateral aspect of the tricuspid annulus.

The cine loops for assessment of peak longitudinal 2D strain of the right ventricle were recorded in apical 4-chamber views and optimised by changing the transducer scan width to achieve a frame rate of at least 40/s. Further analysis was conducted with dedicated, commercial software (EchoPac, GE Vingmed). The speckle tracking analysis consisted of marking the endocardium, defining the width of the region of interest, reflecting the mean distance from endocardium to pericardium and using the analysis software package.

Peak 2D longitudinal strain (LS) and strain rate were defined as the peak negative value on the strain curve during the entire cardiac cycle. The global LS and strain rate value for the RV was calculated as a mean value for the basal and middle segment of the free RV wall. Figure 1 shows an example of echocardiographic measurements of RV function.

\section{Statistical analysis}

All values are presented as mean $\pm \mathrm{SD}$. Comparisons between groups were made using two-tailed Student t test or Welch test and non-parametric methods depending on data distribution. The change of RV function over time in the sAVR compared with the TAVI group was evaluated using an ANOVA test for the interaction effect of time-point and treatment group.

Regression analysis was used to assess the relation of independent variables to the change in the RV function parameters. Statistical analyses were performed using R V.2.13.0 (R Foundation for Statistical Computing, Vienna, Austria) and MedCalc 11.3.3.0 Software (Mariakerke, Belgium).

\section{RESULTS}

\section{Patient characteristics and postprocedural status}

Baseline characteristics are presented in table 1. As expected, patient groups differed significantly. Patients in the TAVI group were older ( $81 \pm 11$ vs $71 \pm 12$ years, $p=0.0004$ ) compared with the sAVR group and presented with more pronounced symptoms. None of them was in New York Heart Association Functional Class (NYHA) I, and $17 \%$ presented with symptoms at rest (NYHA IV). There was a trend towards more patients with previous cardiac surgery and renal impairment (Creatinine $>200 \mu \mathrm{mol} / \mathrm{l})$ in the TAVI compared with the sAVR group $(25 \%$ vs $5 \%$ and $31 \%$ vs $9 \%$, respectively; $\mathrm{p}=0.07$ for both) and the perioperative risk estimated by EuroSCORE was markedly higher in the TAVI than in the aortic valve replacement (AVR) group $(24.3 \pm 14.6 \%$ vs $7.2 \pm 4.7 \%, p<0.0001)$.

In the sAVR group, two patients received a mechanical aortic valve prosthesis (St. Jude Medical bileaflet valve), while the remaining patients received bioprostheses (Carpentier Edwards bioprosthesis, 4; Carpentier Edwards Perimount Bioprosthesis, 16). In this group, 19 patients underwent isolated AVR (four with aortic surgery), while three patients required additional coronary artery bypass graft surgery. The surgical access was via a conventional median sternotomy in all patients. Patients were operated in mild hypothermia (core temperature being 33.1, range $28.0-36.9^{\circ} \mathrm{C}$ ) and all received retrograde blood cardioplegia. The cross-clamp time was $66.8 \pm 18.7 \mathrm{~min}$ and cardiopulmonary bypass time $110.8 \pm 29.6 \mathrm{~min}$. There were no significant differences in the cross-clamp time and cardiopulmonary bypass time between patients with isolated AVR and those with additional procedures. All surgical patients had a favourable postoperative course without any serious 30-day complications.
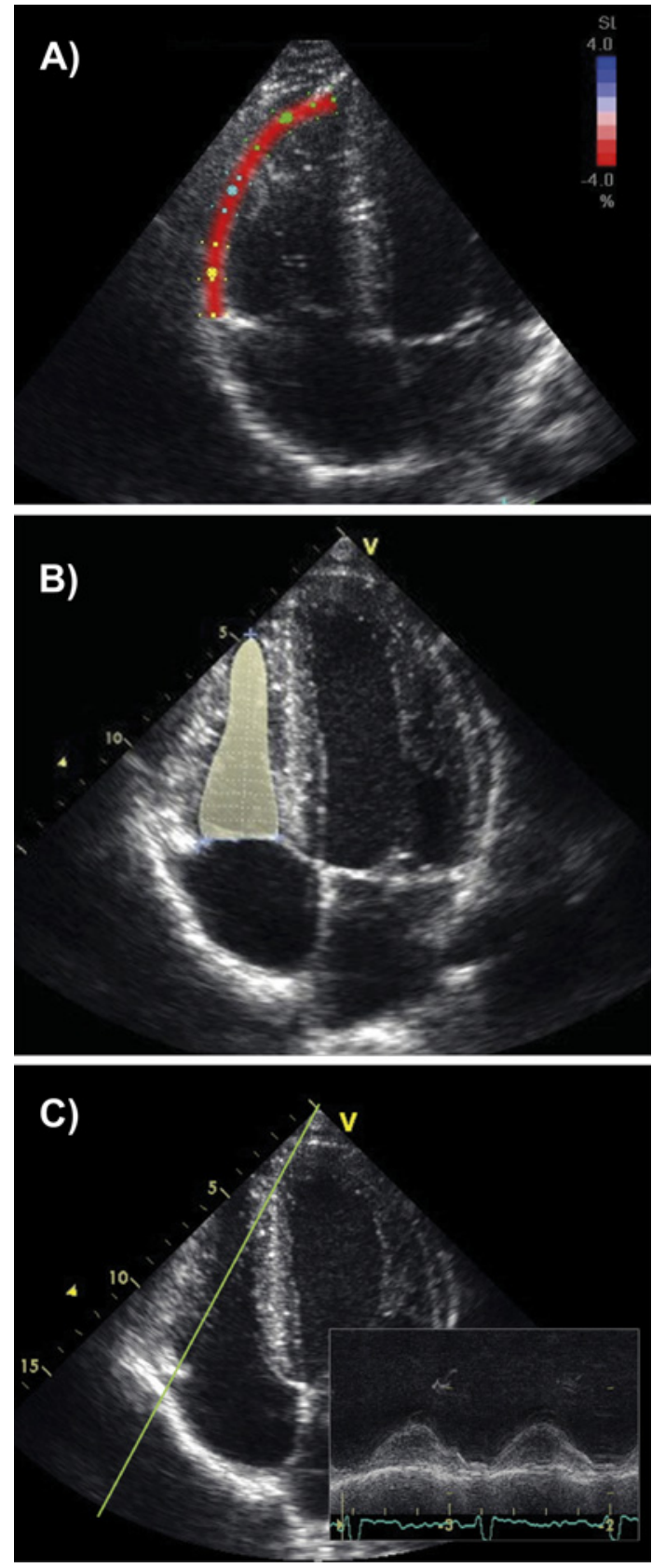

Figure 1 Example of echocardiographic measurements of right ventricle (RV) function including $2 D$ longitudinal $R V$ strain $(A)$, fractional shortening (B) and tricuspid annular plane systolic excursion (C).

In the TAVI group, the majority (60 patients) underwent transfemoral aortic valve implantation while 41 patients had limited vascular access and required a transapical approach. Overall, 95 patients received an Edwards Sapien valve, while six patients underwent implantation of an Edwards Sapien XT valve. The 30 -day mortality was $10 \%$ and was due to bleeding, sepsis, stroke, aortic haematoma, endocarditis, multiple organ failure or pneumonia in two, two, two, one, one, one and one patients, respectively. Strokes occurred in $5 \%$ and $6 \%$ of the patients required a pacemaker implantation. There was a significant difference in the prevalence of clinically relevant pleural effusion between groups ( $32 \%$ in sAVR group vs $10 \%$ in TAVI group, $p=0.018$ ). This may, at least in part, be related to RV dysfunction. 
Table 1 Baseline characteristics of the transcatheter aortic valve implantation (TAVI) and surgical aortic valve replacement (sAVR) group

\begin{tabular}{|c|c|c|c|}
\hline Parameter & TAVI $(n=101)$ & sAVR $(n=22)$ & p Value \\
\hline \multirow[t]{2}{*}{ Age (years) } & $81 \pm 11$ & $71 \pm 12$ & 0.0004 \\
\hline & 82 (IOR 77-86) & 73 (IOR 63-79) & \\
\hline Gender. female (n/\%) & $68 / 67 \%$ & $14 / 63 \%$ & 0.93 \\
\hline \multirow[t]{2}{*}{ Weight $(\mathrm{kg})$} & $75 \pm 17$ & $70 \pm 19$ & 0.3 \\
\hline & 74 (IOR 62-85) & 72 (IOR 65-85) & \\
\hline \multirow[t]{2}{*}{ Height $(\mathrm{cm})$} & $167 \pm 9$ & $168 \pm 10$ & 0.65 \\
\hline & 167 (IOR 160-170) & 166 (IOR 160-173) & \\
\hline NYHA FC (n) (I/II/III/IV) & $0 / 19 / 65 / 17$ & $1 / 4 / 17 / 0$ & 0.033 \\
\hline Logistic euroSCORE (\%) & $24.3 \pm 14.6$ & $7.2 \pm 4.7$ & $<0.0001$ \\
\hline Coronary heart disease $(n / \%)$ & $51 / 50 \%$ & $9 / 41 \%$ & 0.56 \\
\hline Previous cardiac surgery (n/\%) & $25 / 25 \%$ & $1 / 5 \%$ & 0.07 \\
\hline Prior myocardial infarction $(\mathrm{n} / \%)$ & $25 / 25 \%$ & $2 / 9 \%$ & 0.19 \\
\hline Arterial hypertension ( $\mathrm{n} / \%$ ) & $55 / 54 \%$ & $16 / 73 \%$ & 0.18 \\
\hline Diabetes (n/\%) & $22 / 22 \%$ & $3 / 14 \%$ & 0.57 \\
\hline Peripheral artery occlusive disease $(n / \%)$ & $26 / 26 \%$ & $4 / 18 \%$ & 0.64 \\
\hline Chronic renal insufficiency $(\mathrm{n} / \%)$ & $31 / 31 \%$ & $2 / 9 \%$ & 0.07 \\
\hline COPD (n/\%) & $26 / 26 \%$ & $4 / 18 \%$ & 0.64 \\
\hline \multirow[t]{2}{*}{ PCWP (mm Hg) } & $15.6 \pm 11.1$ & $17.9 \pm 7.9$ & 0.42 \\
\hline & 14.0 (IOR 7.7-22.2) & $16.0($ IOR $12.2-24.0)$ & \\
\hline \multirow[t]{2}{*}{ PAP mean $(\mathrm{mm} \mathrm{Hg})$} & $28.0 \pm 11.5$ & $24.4 \pm 9.5$ & 0.29 \\
\hline & $25.5(\operatorname{IQR} 17.0-40.0)$ & 16.2 (IOR 16.2-31.0) & \\
\hline \multirow[t]{2}{*}{ PAP peak $(\mathrm{mm} \mathrm{Hg})$} & $44.5 \pm 18.0$ & $42.9 \pm 19.3$ & 0.76 \\
\hline & $39.0($ IQR 30.0-59.0) & 38.0 (IOR 28.7-48.2) & \\
\hline
\end{tabular}

\section{Transthoracic echocardiography and speckle tracking analysis}

Echocardiographic data are shown in table 2. Aortic valve area before TAVI and sAVR was similar with $0.61 \pm 0.20 \mathrm{~cm}^{2}$ and $0.73 \pm 0.24 \mathrm{~cm}^{2}(p=0.7)$. The valve area reached after intervention was also comparable $\left(1.60 \pm 0.34 \mathrm{~cm}^{2}\right.$ vs $1.60 \pm 0.44 \mathrm{~cm}^{2}$, $\mathrm{p}=1.0)$.

\section{Impact of intervention on RV size and function}

Preinterventional RV size did not significantly differ between TAVI and sAVR patients (table 2). End diastolic RV inflow diameters increased in the sAVR group postoperatively compared with preoperative values $(41.7 \pm 4.1$ vs $39.3 \pm 4.7 \mathrm{~mm}$; $\mathrm{p}=0.048)$, while the end diastolic RV area did not change significantly $\left(17.2 \pm 4.3\right.$ vs $\left.16.3 \pm 3.4 \mathrm{~cm}^{2} ; p=0.31\right)$. In the TAVI group, RV inflow diameters and end diastolic RV area remained unchanged after valve implantation compared with preinterventional values.

RV systolic pressure, as measured invasively, was similar in the TAVI and sAVR groups (46.5 \pm 16.7 vs $44.7 \pm 19.7 \mathrm{~mm} \mathrm{Hg}$; $p=0.7$ ), suggesting that both groups had similar degrees of secondary pulmonary hypertension.

Baseline RV systolic function was significantly better in the sAVR compared with the TAVI group based on TAPSE and RVLS measurements $(24.1 \pm 5.0 \mathrm{~mm}$ vs $15.9 \pm 4.7 \mathrm{~mm}, \mathrm{p}<0.0001$ and $-25.2 \pm 6.1 \%$ vs $-21.3 \pm 5.8 \%, p=0.006$, respectively). After TAVI, however, these parameters did not change significantly (table 2 and figure 2), while a marked deterioration in TAPSE $(24.1 \pm 0.5 .0$ vs $15.9 \pm 4.1 \mathrm{~cm} ; \mathrm{p}<0.0001)$, RV-LS $(-25.2 \pm 6.1 \mathrm{vs}$ $-20.0 \pm 7.0 \% ; p=0.009)$ and RV fractional area change (FAC) (47.0 \pm 7.0 vs $39.8 \pm 10.7 \% ; p=0.019)$ was found in the sAVR group. Considering a reduced RV function with a TAPSE or FAC below $16 \mathrm{~mm}$ or $35 \%$, respectively, there were three patients $(14 \%)$ before but 13 patients $(62 \%)$ after the surgery who had impaired RV function in the sAVR group. The change in none of the parameters of RV function correlated with the cross-clamp and bypass time. There was no significant deterioration of any parameter of RV function after TAVI neither in patients undergoing transapical nor transfemoral TAVI (figure 2).

Paired $t$ test showed a significant reduction in RV function in patients undergoing sAVR while no such effect was seen in the TAVI group (figure 2)

The ANOVA test for the interaction effect of time point (pretreatment; $0-2,2-4,4-6$ and $>6$ months post-treatment) and group (sAVR-TAVI) confirmed that the change of RV function over time was significantly different for sAVR compared with TAVI ( $p$ value for the interaction effect 0.0016 and 0.007 for TAPSE and RV-LS, respectively).

In all, $42 \%$ of patients in the surgical group had a nondominant right coronary artery (RCA) on preoperative coronary angiography. There was no significant difference in postoperative RV function between this group and patients with a dominant RCA ( $p=0.77, p=0.61, p=0.98$ for TAPSE, FAC and RV-LS, respectively).

On the univariate regression analysis including all categorical parameters used for EuroSCORE calculation and study group assignment, only the latter was found to be significantly related to the change in RV-LS and TAPSE $\left(R^{2}=0.17, p=0.002\right.$ and $\mathrm{R}^{2}=0.34, \mathrm{p}<0.0001$, respectively). On this analysis, however, there was no significant relation of assignment of study group to the change in RV-FAC $\left(\mathrm{R}^{2}=0.12, \mathrm{p}=0.056\right)$.

\section{DISCUSSION}

This study evaluated the possibly different effects of TAVI and sAVR on RV function. Including novel parameters of intrinsic myocardial function, we found a significant deterioration in RV function after conventional AVR, while RV function was largely unaffected by TAVI. This difference between the groups 
Table 2 Echocardiographic data for the transcatheter aortic valve implantation (TAVI) and surgical aortic valve replacement (sAVR) group before and after the procedure

\begin{tabular}{|c|c|c|c|c|c|c|}
\hline \multirow[b]{2}{*}{ Parameter } & \multicolumn{2}{|l|}{ TAVI } & \multirow[b]{2}{*}{ p Value } & \multicolumn{2}{|l|}{ SAVR } & \multirow[b]{2}{*}{ p Value } \\
\hline & Pre & Post & & Pre & Post & \\
\hline \multirow[t]{2}{*}{ AoV peak gradient $(\mathrm{mm} \mathrm{Hg})$} & $83.1 \pm 26.2$ & $19.0 \pm 6.6$ & $<0.0001$ & $97.9 \pm 26.6$ & $26.3 \pm 8.8$ & $<0.0001$ \\
\hline & $78.6(60.8-92.0)$ & $18.6(14.4-22.3)$ & & $89.2(80.5-113.8)$ & $23.1(18.8-36.1)$ & \\
\hline AoV mean gradient $(\mathrm{mm} \mathrm{Hg})$ & $50.3(40.5-63.6)$ & $10.0(8.4-12.6)$ & & $60.0(55.2-75.8)$ & $13.4(12.0-18.7)$ & \\
\hline \multirow[t]{2}{*}{ AVA $\left(\mathrm{cm}^{2}\right)$} & $0.61 \pm 0.20$ & $1.60 \pm 0.34$ & $<0.0001$ & $0.73 \pm 0.24$ & $1.60 \pm 0.44$ & 0.006 \\
\hline & $0.64(0.47-0.75)$ & $1.60(1.37-1.79)$ & & $0.65(0.49-0.73)$ & $1.27(0.92-1.9)$ & \\
\hline \multirow[t]{2}{*}{ IVSd (mm) } & $15.4 \pm 2.8$ & $14.7 \pm 2.9$ & 0.23 & $14.9 \pm 1.7$ & $14.3 \pm 2.2$ & 0.09 \\
\hline & $15.5(13.6-17.2)$ & $14.3(13.0-17.0)$ & & $15.0(14.0-16.0)$ & $14.0(13.0-15.0)$ & \\
\hline \multirow[t]{2}{*}{ LV EF (\%) } & $56.7 \pm 17.3$ & $57.5 \pm 15.8$ & 0.59 & $67.7 \pm 7.7$ & $68.2 \pm 7.3$ & 0.84 \\
\hline & $58.6(44.9-68.5)$ & $59.4(50.0-69.4)$ & & $69.4(63.2-73.3)$ & $69.1(61.5-72.5)$ & \\
\hline RV EDD $(\mathrm{mm})$ & $37.7 \pm 6.2$ & $39.2 \pm 9.9$ & 0.29 & $39.3 \pm 4.7$ & $41.7 \pm 4.1$ & 0.048 \\
\hline RV FAC $(\%)$ & $40.8(32.6-49.4)$ & $41.4(34.6-47.2)$ & & $46.4(39.6-49.275)$ & $37.4(31.5-46.4)$ & \\
\hline \multirow[t]{2}{*}{ RV-LS (\%) } & $21.3 \pm 5.8$ & $23.7 \pm 7.7$ & 0.1 & $25.2 \pm 6.1$ & $20.0 \pm 7.0$ & 0.009 \\
\hline & $20.6(18.0-24.6)$ & $23.7(19.9-26.1)$ & & $31.6(21.9-31.6)$ & $21.6(15.8-24.9)$ & \\
\hline \multirow[t]{2}{*}{ TASPE (mm) } & $15.9 \pm 4.7$ & $16.6 \pm 4.7$ & 0.2 & $24.1 \pm 5.0$ & $15.9 \pm 4.1$ & $<0.0001$ \\
\hline & $16.8(13.2-19.9)$ & $17.8(13.3-20.5)$ & & $23.0(20.7-28.0)$ & $17.0(12.7-19.4)$ & \\
\hline \multirow[t]{2}{*}{$\operatorname{RVSP}(\mathrm{mm} \mathrm{Hg})$} & $47.2 \pm 11.9$ & $42.1 \pm 10.0$ & 0.009 & $40.7 \pm 9.2$ & $32.0 \pm 7.6$ & 0.06 \\
\hline & $44.6(38.8-54.9)$ & $48.5(37.8-55.4)$ & & $41.6(36.4-44.7)$ & $34.9(34.6-41.3)$ & \\
\hline
\end{tabular}

Data, if appropriate, are presented as mean \pm SD and median and IQR. Significant values are printed in bold.

AoV, aortic valve; AVA, aortic valve area; EDA, end diastolic area; EDD, end diastolic diameter; EF, ejection fraction; FAC, fractional area change; IVSd, interventricular septum diastolic thickness; LS, absolute value of 2D peak longitudinal strain; LV, left ventricle; RV, right ventricle; RVSP, RV systolic pressure by echocardiography; TAPSE, tricuspid annular plane systolic excursion.

persisted after accounting for the different baseline risk profile. The deterioration in RV function as a consequence of cardiac surgery in general is consistent with the results of previous surgical studies. ${ }^{16-18}$ RV function and its changes with TAVI have so far not been studied in detail in larger patient populations. Although reporting only very small patient groups $(\leq 20$ patients) and providing limited information on RV function (TAPSE only in one study and largely angle dependent tissue Doppler measurements in the other study), two very recent publications support the finding that TAVI-in contrast to sAVR-does not negatively affect RV function. ${ }^{19}{ }^{20}$ Pericardiotomy that has been reported to result in perturbation of myocardial blood flow in the right ventricle and to negatively affect RV end diastolic pressure could be one explanation. ${ }^{21} 22$ The systemic inflammatory response due to the release of proinflammatory cytokines in association with cardiopulmonary bypass could be another reason for the damage of RV myocardium. ${ }^{23}$ A recent intraoperative study, however, has highlighted that RV myocardial function declines almost immediately after the opening of the pericardium, suggesting that cardiopulmonary bypass is not the major cause of the observed deterioration in RV function. ${ }^{24}$ Instead, loss of the pericardial support and the resulting increase in transmural pressure with increased wall stress, which could injure the myocardium or make it susceptible to a change in behaviours when the pericardial constraint is lost, may be the predominant mechanism for this finding. The lack of a detrimental effect of TAVI on the RV may be especially important for high operative risk patients as the development of postoperative RV dysfunction in high risk patients has been reported to be associated with increased morbidity and mortality. ${ }^{2526}$ To date, baseline RV function is not routinely considered when selecting patients for TAVI or sAVR. The results of the current study suggest that in patients with preexisting RV dysfunction, sAVR could lead to further deterioration of RV function, while no further deterioration is to be expected with TAVI. Interestingly, no deterioration in RV function was found in TAVI patients who underwent transapical valve implantation suggesting that while extensive pericardiotomy to expose the heart fully adversely affects RV function, the limited pericardiotomy required for transapical TAVI does not have the same negative impact on RV function.

In addition, pulmonary hypertension and depressed RV function increase the risk of non-cardiac surgery. Even for TAVI, therefore, procedural risk may be increased in this setting. ${ }^{27} 28$

\section{Limitations of the study}

The follow-up time of approximately 3 months in our study was relatively short. Further studies with a longer follow-up period are required to assess the long-term impact of TAVI and sAVR on the RV function. It is also unclear at present how much impact on outcome the different effects of sAVR and TAVI on RV function have.

The differences in baseline characteristics may be considered as a limitation of this study in general. However, the fact that RV function was not affected in TAVI patients despite their significantly worse baseline characteristics makes it highly unlikely that the different effects of TAVI versus sAVR on RV function should not be present in patient groups with comparable baseline characteristics.

We cannot completely exclude the possibility that sAVR patients who underwent repeat postsurgical echocardiograms and were included in the study represent a biased subgroup with 
Patients undergoing AVR

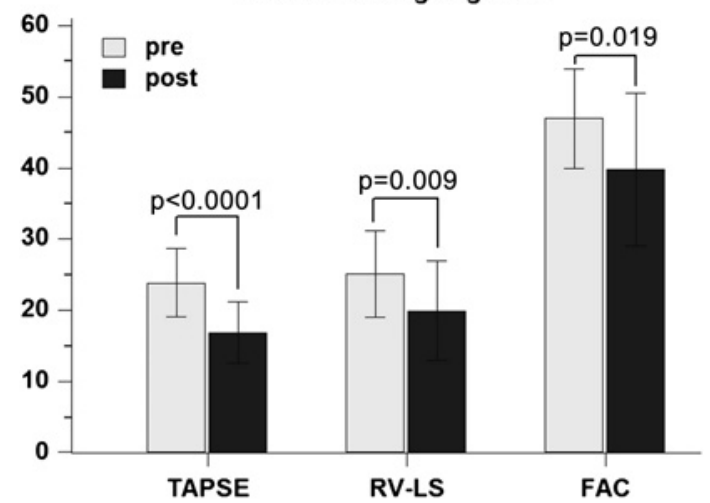

Patients undergoing transapical TAVI

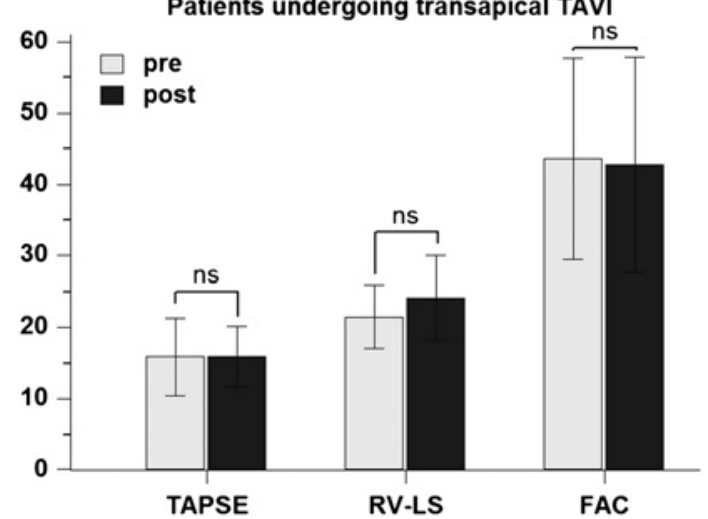

Patients undergoing transfemoral TAVI

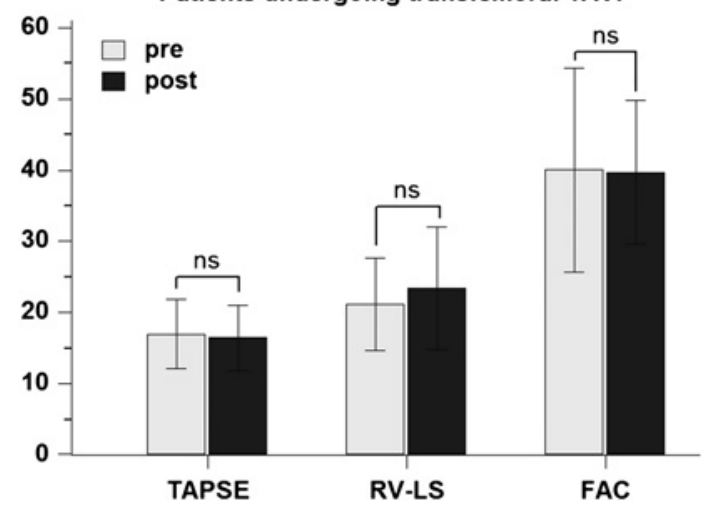

Figure 2 Changes in the right ventricle (RV) function parameters in patients undergoing surgical aortic valve replacement (AVR), transapical transcatheter aortic valve implantation (TAVI) and transfemoral TAVI. TAPSE, tricuspid annular plane systolic excursion (mm); RV-LS, RV- longitudinal strain, absolute value of the global RV-LS (\%); FAC, RV fractional area change (\%); $p$ values relate to paired $t$ tests between groups.

a higher risk profile. We do not, however, operate a policy to determine timing of echocardiographic reassessment based on preoperative risk profile.

The TAVI cohort which consists of two subgroups is significantly larger than the sAVR group included. This may be regarded as a major limitation of the study; however, the study results are significant with the given group size and it is unlikely that including more sAVR patients would relevantly alter the results of the study.

The follow-up time in our study was limited. Furthermore, this is primarily an echocardiographic study and we were not

\section{Key messages}

While transcatheter aortic valve implantation did not affect right ventricular (RV) function, it deteriorated significantly in patients undergoing surgical aortic valve replacement. We speculate that this may be related to the detrimental effects of pericardiotomy and to a lesser degree to cardiopulmonary bypass. While further studies are required to assess the clinical significance of this finding, these data suggest that patients with pre-existing RV dysfunction may benefit from transcatheter aortic valve implantation and that RV function should be incorporated into future risk scores.

able to demonstrate differences in clinical outcome between both subgroups that could be related to differences in RV function. A potential impact on clinical outcome needs to be evaluated in a larger prospective trial with longer follow-up.

\section{CONCLUSIONS}

While RV function is not affected by TAVI, it appears to deteriorate at least temporarily after sAVR. We speculate that this may be related to the detrimental effects of pericardiotomy and to a lesser degree to cardiopulmonary bypass. While further studies are required to assess the clinical significance of this finding, these data suggest that patients with pre-existing RV dysfunction may benefit from TAVI and that RV function should be incorporated into future risk scores.

Contributors All authors have read and approved the manuscript. All authors have also sufficiently contributed to conception and design of the study, analysis and interpretation of data, drafting of the manuscript or revising it to justify authorship.

Funding The Adult Congenital and Valvular Heart Disease Center, University Hospital of Muenster has received support by the EMAH Foundation Karla Voellm, Krefeld, Germany. AK was supported by the Deutsche Herzstiftung e.V.

Competing interests $\mathrm{HB}$ acts as a consultant and proctor for 'Edwards Lifesciences', all the other authors have no disclosures. For HB, the ICMJE form will be uploaded.

Ethics approval The ethics approval was provided by the Research Ethics Committee, University of Muenster, Germany.

Provenance and peer review Not commissioned; internally peer reviewed.

\section{REFERENCES}

1. Piazza N, Wenaweser $\mathrm{P}$, van Gameren $\mathrm{M}$, et al. Relationship between the logistic EuroSCORE and the Society of Thoracic Surgeons Predicted risk of mortality score in patients implanted with the CoreValve ReValving system-a Bern-Rotterdam study. Am Heart J 2010;159:323-9.

2. O'Brien SM, Shahian DM, Filardo G, et al. The Society of Thoracic Surgeons 2008 cardiac surgery risk models: part 2-isolated valve surgery. Ann Thorac Surg 2009;88 (Suppl 1):S23-42.

3. Roques F, Nashef SA, Michel P, et al. Risk factors and outcome in European cardiac surgery: analysis of the EuroSCORE multinational database of 19030 patients. Eur $J$ Cardiothorac Surg 1999;15:816-22; discussion 22-3.

4. Thomas M, Schymik G, Walther T, et al. Thirty-day results of the SAPIEN aortic Bioprosthesis European Outcome (SOURCE) Registry: a European registry of transcatheter aortic valve implantation using the Edwards SAPIEN valve. Circulation 2010;122:62-9.

5. Robiolio PA, Rigolin VH, Hearne SE, et al. Left ventricular performance improves late after aortic valve replacement in patients with aortic stenosis and reduced ejection fraction. Am J Cardiol 1995;76:612-15.

6. Giorgi D, Di Bello V, Talini E, et al. Myocardial function in severe aortic stenosis before and after aortic valve replacement: a Doppler tissue imaging study. J Am Soc Echocardiogr 2005;18:8-14.

7. Rost C, Korder S, Wasmeier G, et al. Sequential changes in myocardial function after valve replacement for aortic stenosis by speckle tracking echocardiography. Eur $\mathrm{J}$ Echocardiogr 2010;11:584-9.

8. Biederman RW, Doyle M, Yamrozik J, et al. Physiologic compensation is supranormal in compensated aortic stenosis: does it return to normal after aortic valve replacement 
or is it blunted by coexistent coronary artery disease? An intramyocardial magnetic resonance imaging study. Circulation 2005;112(Suppl 9):1429-36.

9. Kurnik PB, Innerfield M, Wachspress JD, et al. Left ventricular mass regression after aortic valve replacement measured by ultrafast computed tomography. Am Heart $J$ 1990; 120:919-27

10. Haddad F, Couture $P$, Tousignant $C$, et al. The right ventricle in cardiac surgery, a perioperative perspective: I. Anatomy, physiology, and assessment. Anesth Analg 2009;108:407-21

11. Haddad F, Couture $\mathrm{P}$, Tousignant $\mathrm{C}$, et al. The right ventricle in cardiac surgery, a perioperative perspective: II. Pathophysiology, clinical importance, and management. Anesth Analg 2009;108:422-33.

12. Shahian DM, O'Brien SM, Filardo G, et al; Society of Thoracic Surgeons Quality Measurement Task Force. The Society of Thoracic Surgeons 2008 cardiac surgery risk models: part 3-valve plus coronary artery bypass grafting surgery. Ann Thorac Surg 2009;88(Suppl 1):S43-62.

13. Lang RM, Bierig M, Devereux RB, et al; American Society of Echocardiography's Nomenclature and Standards Committee; Task Force on Chamber Quantification; American College of Cardiology Echocardiography Committee; American Hear Association; European Association of Echocardiography, European Society of Cardiology. Recommendations for chamber quantification. Eur J Echocardiogr 2006; 7:79-108.

14. Gardin JM, Adams DB, Douglas PS, et al. Recommendations for a standardized report for adult transthoracic echocardiography: a report from the American Society of Echocardiography's Nomenclature and standards Committee and Task Force for a Standardized echocardiography report. J Am Soc Echocardiogr 2002:15:275-90.

15. Rudski LG, Lai WW, Afilalo J, et al. Guidelines for the echocardiographic assessment of the right heart in adults: a report from the American Society of echocardiography endorsed by the European association of echocardiography, a registered branch of the European Society of Cardiology, and the Canadian Society of echocardiography. J Am Soc Echocardiogr 2010;23:685-713; quiz 86-8.

16. Hedman A, Alam M, Zuber E, et al. Decreased right ventricular function after coronary artery bypass grafting and its relation to exercise capacity: a tricuspid annular motion-based study. J Am Soc Echocardiogr 2004;17:126-31.
17. Alam M, Hedman A, Nordlander R, et al. Right ventricular function before and after an uncomplicated coronary artery bypass graft as assessed by pulsed wave Doppler tissue imaging of the tricuspid annulus. Am Heart $J$ 2003;146:520-6.

18. Diller GP, Wasan BS, Kyriacou A, et al. Effect of coronary artery bypass surgery on myocardial function as assessed by tissue Doppler echocardiography. Eur $\mathrm{J}$ Cardiothorac Surg 2008:34:995-9.

19. Zhao Y, Lindqvist P, Nilsson J, et al. Trans-catheter aortic valve implantation-early recovery of left and preservation of right ventricular function. Interact Cardiovasc Thorac Surg 2011:12:35-9.

20. Forsberg LM, Tamas E, Vanky F, et al. Left and right ventricular function in aortic stenosis patients 8 weeks post-transcatheter aortic valve implantation or surgical aortic valve replacement. Eur J Echocardiogr 2011;12:603-11.

21. Schosser R, Forst $H$, Racenberg J, et al. Open chest and open pericardium affect the distribution of myocardial blood flow in the right ventricle. Basic Res Cardiol 1990:85:508-18.

22. Assanelli D, Lew WY, Shabetai R, et al. Influence of the pericardium on right and left ventricular filling in the dog. J Appl Physiol 1987:63:1025-32.

23. Davidson MJ. Can the off-pump coronary artery bypass debate shed light on postoperative right heart dysfunction? Circulation 2008;117:2181-3.

24. Unsworth B, Casula RP, Kyriacou AA, et al. The right ventricular annular velocity reduction caused by coronary artery bypass graft surgery occurs at the moment of pericardial incision. Am Heart J 2010;159:314-22.

25. Boldt J, Zickmann B, Ballesteros $\mathrm{M}$, et al. Right ventricular function in patients with aortic stenosis undergoing aortic valve replacement. J Cardiothorac Vasc Anesth 1992;6:287-91.

26. Derumeaux G, Ovize M, Loufoua J, et al. Doppler tissue imaging quantitates regional wall motion during myocardial ischemia and reperfusion. Circulation 1998:97:1970-7.

27. Memtsoudis SG, Ma Y, Chiu YL, et al. Perioperative mortality in patients with pulmonary hypertension undergoing major joint replacement. Anesth Analg 2010:111:1110-16.

28. Ramakrishna G, Sprung J, Ravi BS, et al. Impact of pulmonary hypertension on the outcomes of noncardiac surgery: predictors of perioperative morbidity and mortality. J Am Coll Cardiol 2005:45:1691-9. 